\title{
Energy-Efficient Data Acquisition in Mobile Crowdsensing Systems
}

\author{
Andrea Capponi \\ FSTC-CSC, University of Luxembourg, Luxembourg, E-mail: andrea.capponi@uni.lu
}

\begin{abstract}
Mobile Crowdsensing (MCS) is one of the most promising paradigms for monitoring phenomena in urban environments. The success of a MCS campaign relies on large participation of citizens, who may be reluctant in joining a campaign due to sensing and reporting costs they sustain. Hence, it is fundamental to propose efficient data collection frameworks (DCFs). In the first stages of our work, we proposed an energyefficient DCF that aims to minimize energy consumption while maximizing the utility of contributed data. Then, we developed an Android application and proposed a methodology to compare several DCFs, performing energy- and network-related measures with Power Monitor and Wireshark. Currently, we are investigating collaborative data delivery as a more efficient solution than the individual one. The key idea is to form groups of users and elect a responsible for aggregated data delivery. To this end, it is crucial to analyze device to device (D2D) communications and propose efficient policies for group formation and owner election. To evaluate the performance in realistic urban environments we exploit CrowdSenSim, which runs large-scale simulations in citywide scenarios.
\end{abstract}

\section{INTRODUCTION}

In the last years, the unprecedented growth of population living in cities calls for a sustainable urban development. Sensing is fundamental to monitor the current status of infrastructures and the resource utilization [1]. Deploying sensing infrastructures is typically expensive, while including citizens in the loop through mobile crowdsensing (MCS) has been proven as a win-win strategy [2]. Indeed, it allows to exploit already deployed infrastructures with no need of further investments. Mobility and intelligence of human participants guarantee higher coverage and better context awareness, if compared to traditional sensor networks [3]. In addition, users ensure self-maintenance and recharge of the devices that act as sensor and communication nodes and are mobile, unprecedentely from other paradigms. Smartphones are equipped with a rich set of sensors suitable in multiple domains, such as environmental monitoring, health care and public safety. Available communication technologies deliver the acquired data to a collector, typically located in the cloud, for data processing and analysis. The success of MCS campaigns relies on large participation of citizens, who sustain costs (e.g., the battery drain of their devices) to contribute data. For this reason, it is fundamental to devise efficient data collection frameworks (DCFs). To this end, we developed an energy-efficient DCF and proposed a novel methodology to evaluate performance of several DCFs [4]. MCS systems typically employ users to collect data individually, but collaborative sensing represents an

978-1-5386-4725-7/18/\$31.00 @2018 IEEE efficient solution to save energy. Our aim is to investigate how forming groups of users who exploit D2D communications to exchange data within each group and how to perform election of a group owner, who is responsible to report data to the central collector. MCS systems require a large participation of users to be effective and testbeds are often not feasible. Hence, we specifically designed a custom simulator to evaluate the performance of MCS activities in realistic urban scenario and we present results obtained through large-scale simulations [5].

\section{BACKGROUND}

This Section briefly introduces three general-purpose DCFs, which represent different families of methodologies to efficiently acquire information. Other existing DCFs in literature present minor variations with respect to the considered ones. These DCFs are opportunistic MCS systems, which are based on a minimal intervention from users as sensing decisions are application- or device-driven. The first considered DCF is a deterministic distributed framework (DDF) [6] we proposed to maximize the utility of acquired data, while minimizing the energy consumption for sensing and delivery. Piggyback CrowdSensing (PCS) [7] is a DCF that consistently lowers energy consumption associated to reporting operations performing data delivery during the so called smartphone opportunities (e.g., phone calls). Finally, Montori et al. [8] propose a probabilistic distributed algorithm (PDA) to save energy limiting overhead and data redundancy. The algorithm exploits a feedback from the central authority to set probabilistic thresholds for sensing decisions in each region of interest.

\section{ENERGY-EFFICIENT DATA ACQUISITION IN MCS SYSTEMS}

This section presents our works on energy-efficient MCS systems. First, we introduce a methodology to assess the performance of DCFs. Then, we propose a collaborative sensing that exploits D2D communications to form groups and elect an owner who is the only responsible for data delivery. Finally, we present CrowdSenSim [5], a custom simulator we specifically designed to assess the performance of MCS systems in largescale urban environments.

\section{A. A methodology to profile energy consumption of DCFs}

A MCS campaign requires a large participation of citizens to be effective. They sustain costs for sensing and delivery operations and may be reluctant in joining a campaign or contributing data actively. Hence, it is crucial to devise efficient 


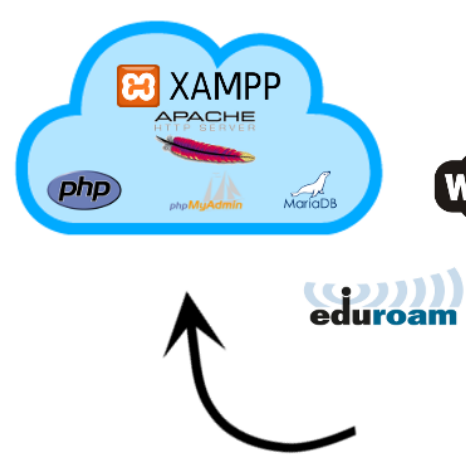

(a) Architecture of the mobile application

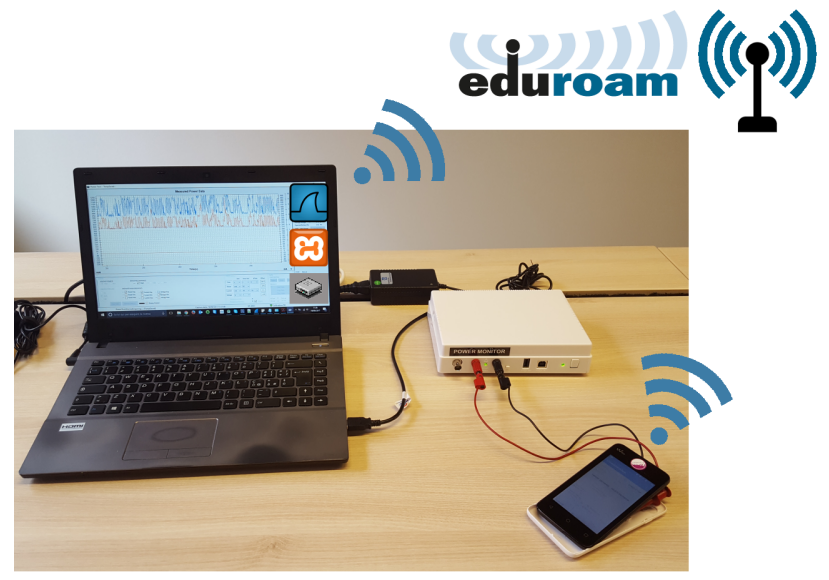

(b) Power measurements setup

Fig. 1. Experimental set-up to perform energy- and network-related measurements

DCFs. To this end, we present a methodology to assess the performance of DCFs and compare the ones discussed in Sec. II, which differ by several aspects that impact their efficiency. First, they exploit different data reporting mechanisms (DRMs), such as continuous (i.e., data is delivered in real time), delayed (i.e., data is sent after the sensing activity) and probabilistic (i.e., data transmission is occasional while sensing). Then, the degree of control the central authority establishes through feedback has a consistent impact on the amount of acquired data and the energy consumption. We developed a custom Android application to emulate DRMs on a mobile device. Fig. 1(a) exhibits our experimental set-up for the Android application. We performed experiments to profile the energy consumption with a Power Monitor and network-related measurements with Wireshark, a free and open source packet analyzer. Fig. 1(b)) shows the set-up for energy- and network-related measurements.

\section{B. Collaborative data delivery}

In MCS campaigns citizens usually contribute data individually and independently from others. This strategy implies a direct task allocation to each user, who should be able to deliver acquired data through available communication technologies, such as WiFi or cellular data interfaces. It is well known that mobile devices consume energy mostly due to data delivery than sensing operations. Hence, leveraging device to device (D2D) communications [9] (e.g., WiFi direct, LTE direct, Bluetooth) within a group of participants that sense the same phenomena in the same area represents a win-win solution. Collaborative data delivery is based on grouping users that exchange data within the group and electing an owner, who is the only responsible to send the aggregated data to the central collector [10]. Hence, it is fundamental to propose strategies to form groups and elect a responsible according to several criteria. We present different policies and exploit WiFi Direct communication technology for performance evaluation. The first policy is called static grouping and consists in forming groups of citizens inside cells of a regular grid that covers the whole area of interest of the campaign. The second policy is the point of interest (POI) grouping, which takes into account users in the surroundings of a place, considering that people typically tend to stay for a certain amount of time in these POIs (e.g., shopping centers or bus stops). The last one is the dynamic grouping, based on the fact that people walking on a street in the same direction tend to group. Owner election takes into account users' position, direction, speed and battery level of device.

\section{CrowdSenSim and simulation results}

As MCS systems require large participation of users to be effective, performing experiments on real testbeds is not often feasible. To this end, simulations are a valid alternative and CrowdSenSim is a custom simulator we specifically designed to assess the performance of crowdsensing activities in large urban areas [5]. CrowdSenSim supports pedestrian mobility in city-wide scenarios and is composed by independent modules representing inputs of the particular MCS campaign (see Fig. 2(a)). Modeling the urban environment with high precision is a key ingredient to obtain effective results and CrowdSenSim provides the street network graph at any desired level of precision (see Fig. 2(b)) through an algorithm running on its background. All the individual walking paths are obtained before simulation runtime to ensure the scalability of the platform. During runtime, users move following their predetermined trajectories and contribute data according to the implemented DCF, exploiting sensors typically available in mobile devices. The simulator computes the amount of gathered data for each user and the associated battery drain for sensing and reporting. After the runtime it is possible to obtain different results, such as heatmaps with the normalized amount of gathered data (see Fig. 2(c)).

Fig. 3 shows the amount of contributed data and the corresponding battery drain for each DCF under analysis. Marks in the graphic represent the battery drain that users have consumed to contribute a certain amount of data. DDF exhibits a low number of marks because mobile devices have a similar behavior due to a stopping mechanism which indirectly controls the energy consumption. On the other side, PDA and PCS show much higher variability due to the different reporting mechanisms (delayed and probabilistic) and their designs: to 


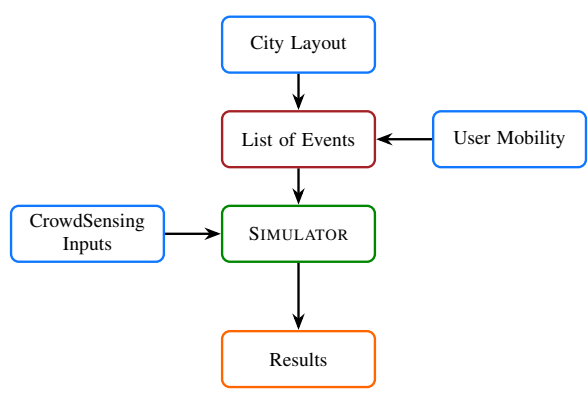

(a) Main modules of CrowdSenSim

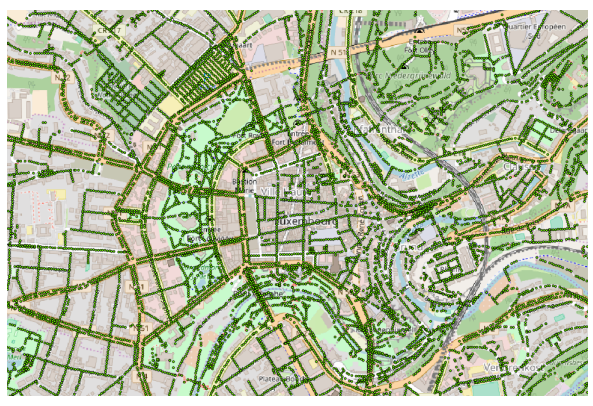

(b) The green dots are lanes where users can walk Fig. 2. Main features of CrowdSenSim

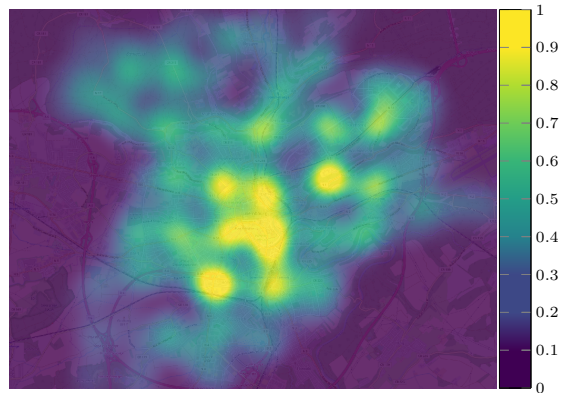

(c) Normalized distribution of contributed data

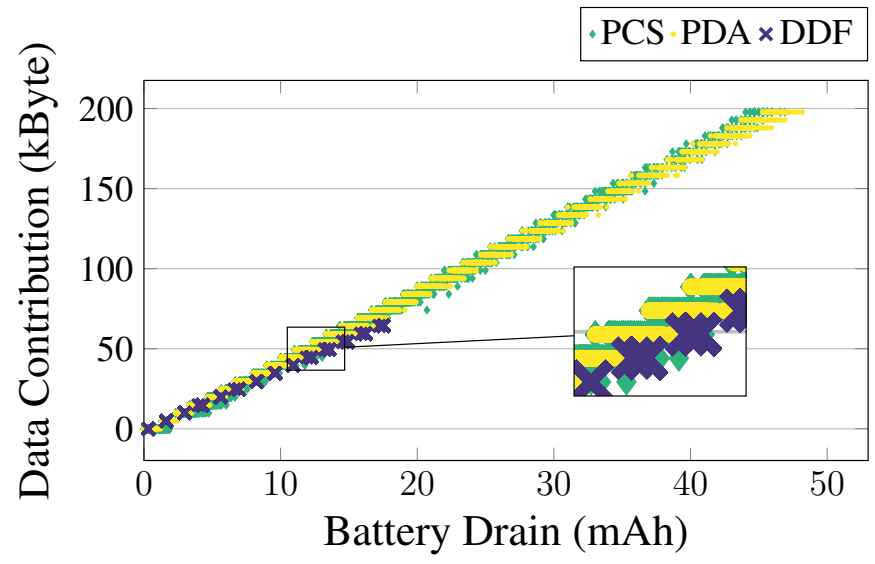

Fig. 3. Amount of collected data and the associated battery drain in Luxembourg City

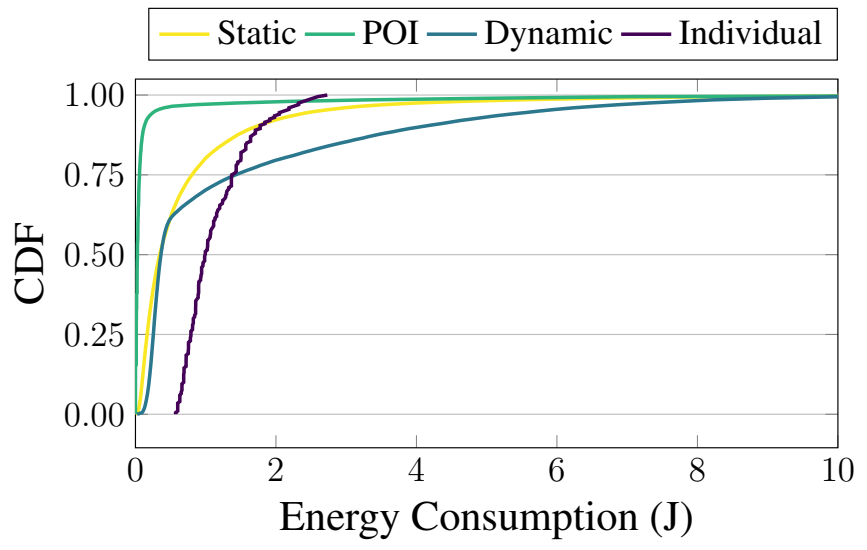

Fig. 4. CDF of total energy consumption for different data collection policies

contribute a certain amount of data, the participants spend different amounts of energy.

Fig. 4 shows preliminary results about collaborative sensing, comparing individual reporting with the three different groupbased policies we have considered. It exhibits that in $75 \%$ of the cases users have a lower consumption exploiting collaborative strategies. They raise until approximately $90 \%$ and almost all of them respectively with static or POI policy.

\section{CONCLUSION}

Large participation of users is essential for the success of a campaign and devising energy-efficient DCFs is fundamental to achieve it. In our early-stage work, we first proposed an energy-efficient DCF that minimizes the costs users sustain, while maximizing the utility of collected information. Then, we developed an Android application that can implement DCFs and we profiled real energy and network measurements exploiting a power monitor and Wireshark. These measures have been included in CrowdSenSim, specifically designed to simulate MCS activities in realistic urban environments. Ongoing work leverages D2D communications to perform collaborative data delivery, which is more energy efficient compared to the individual approach. The idea is based on forming groups of users and electing a group owner, who is the only responsible to report aggregated data to the central collector.

\section{REFERENCES}

[1] G. Cardone, A. Cirri, A. Corradi, L. Foschini, R. Ianniello, and R. Montanari, "Crowdsensing in urban areas for city-scale mass gathering management: Geofencing and activity recognition," IEEE Sensors Journal, vol. 14, no. 12, pp. 4185-4195, Dec 2014.

[2] W. Khan, Y. Xiang, M. Aalsalem, and Q. Arshad, "Mobile phone sensing systems: A survey," IEEE Communications Surveys Tutorials, vol. 15, 2013.

[3] R. Ganti, F. Ye, and H. Lei, "Mobile crowdsensing: current state and future challenges," IEEE Communications Magazine, vol. 49, no. 11, pp. 32-39, November 2011.

[4] M. Tomasoni, A. Capponi, C. Fiandrino, D. Kliazovich, F. Granelli, and P. Bouvry, "Profiling energy efficiency of mobile crowdsensing data collection frameworks for smart city applications," in The 6th IEEE International Conference on Mobile Cloud Computing, Services, and Engineering (IEEE Mobile Cloud 2018), 2018.

[5] C. Fiandrino, A. Capponi, G. Cacciatore, D. Kliazovich, U. Sorger, P. Bouvry, B. Kantarci, F. Granelli, and S. Giordano, "CrowdSenSim: a simulation platform for mobile crowdsensing in realistic urban environments," IEEE Access, vol. 5, pp. 3490-3503, Feb 2017.

[6] A. Capponi, C. Fiandrino, D. Kliazovich, P. Bouvry, and S. Giordano, "A cost-effective distributed framework for data collection in cloud-based mobile crowd sensing architectures," IEEE Transactions on Sustainable Computing, vol. 2, no. 1, pp. 3-16, Jan 2017.

[7] N. D. Lane, Y. Chon, L. Zhou, Y. Zhang, F. Li, D. Kim, G. Ding, F. Zhao, and H. Cha, "Piggyback crowdsensing (PCS): Energy efficient crowdsourcing of mobile sensor data by exploiting smartphone app opportunities," in 11th ACM Conference on Embedded Networked Sensor Systems, ser. SenSys, 2013, pp. 1-14.

[8] F. Montori, L. Bedogni, and L. Bononi, "Distributed data collection control in opportunistic mobile crowdsensing," in Proc. of the 3rd Workshop on Experiences with the Design and Implementation of Smart Objects, ser. SMARTOBJECTS. ACM, 2017, pp. 19-24.

[9] D. Camps-Mur, A. Garcia-Saavedra, and P. Serrano, "Device-to-Device communications with Wi-Fi Direct: overview and experimentation," IEEE Wireless Communications, vol. 20, no. 3, pp. 96-104, June 2013.

[10] C. E. Casetti, C. F. Chiasserini, Y. Duan, P. Giaccone, and A. P. Manriquez, "Data connectivity and smart group formation in Wi-Fi Direct multi-group networks," IEEE Transactions on Network and Service Management, vol. 15, no. 1, pp. 245-259, March 2018. 